ORIGINAL ARTICLE

\title{
Sections of the nipple and quadrants in mastectomy specimens for carcinoma are of limited value
}

\author{
K Sikand, A H S Lee, S E Pinder, C W Elston, I O Ellis
}

J Clin Pathol 2005;58:543-545. doi: 10.1136/icp.2004.022665

See end of article for authors' affiliations .....................

Correspondence to: Dr A H S Lee, Department of Histopathology, City Hospital, Hucknall Road, Nottingham NG5 1PB, UK; alee1@ncht.trent.nhs.uk

Accepted for publication 28 October 2004

\begin{abstract}
Aim: To assess the value of nipple and quadrant sections in mastectomy specimens for carcinoma in detecting Paget's disease and multifocal carcinoma.

Methods: Two hundred and forty eight consecutive mastectomies performed for carcinoma were reviewed. The presence of Paget's disease of the nipple and mode of identification of any multifocal carcinoma was recorded.

Results: Nipple sections showed Paget's disease in eight specimens: in five the diagnosis had been made on previous biopsy and in three (1\%) this was a new diagnosis. In the 220 specimens in which all four quadrants were sampled, multifocal disease was identified more often in specimens with invasive carcinoma (39 of 186; $21 \%$ ) than in those with only ductal carcinoma in situ (0 of 34). In specimens with invasive carcinoma, multifocality was identified macroscopically in 20: on microscopy of tumour sections in four, on microscopic examination of quadrant sections in 11, in the nipple in three, and in both quadrant and nipple sections in one. Overall, multifocality was found on microscopic examination of quadrant or nipple sections in 15 of 220 specimens (7\%).

Conclusions: The low frequency of detection of multifocality or Paget's disease in nipple and quadrant sections from mastectomy specimens, combined with the fact that such findings do not affect patient management, suggest that nipple and quadrant sections should only be taken if resources permit.
\end{abstract}

$M$ ost patients in the UK undergoing mastectomy for carcinoma will have had a preoperative diagnosis based on core needle biopsy or fine needle aspiration cytology. Some will have had previous therapeutic wide local excision and will be undergoing "completion mastectomy"; others may occasionally have had previous diagnostic surgical biopsy. The main purposes of the pathological examination of a mastectomy for carcinoma in patients without previous breast surgery are confirmation of the diagnosis and assessment of prognostic and predictive factors to guide subsequent management. In patients with a preoperative diagnosis of ductal carcinoma in situ (DCIS), the search for invasive carcinoma is also important. Therefore, most sections taken are of the carcinoma. In patients with previous surgery, the biopsy cavity is sampled to search for residual disease. Sections should also be taken of any further suspicious areas.

"The main purposes of the pathological examination of a mastectomy for carcinoma in patients without previous breast surgery are confirmation of the diagnosis and assessment of prognostic and predictive factors to guide subsequent management"

In addition, several guideline documents suggest sampling of the nipple and quadrants. Rosen recommends taking a vertical section of the nipple and two random sections from each quadrant. ${ }^{1}$ The 1995 National Health Service Breast Screening Programme (NHSBSP) guidelines recommend that at least one block should be taken from each quadrant and ideally two from the nipple-one in the sagittal and one in the coronal plane through the junction with the areola. ${ }^{2}$ However, the importance of these additional blocks has recently been questioned, and the 2005 NHSBSP guidelines state that sampling of the nipple and quadrants can be performed if resources permit. ${ }^{3}$
The only previous study of sampling of mastectomy specimens reported that additional information was derived from the quadrant sections in 21 of 78 mastectomy specimens, particularly the identification of multifocal or multicentric disease. ${ }^{4}$ This figure is much higher than we would have expected from our experience in Nottingham. The purpose of our current study was to investigate the value of sampling of the nipple and quadrants from routine mastectomy specimens for primary operable breast carcinoma.

\section{MATERIALS AND METHODS}

The cut up technique used in Nottingham follows the 1995 NHSBSP guidelines. ${ }^{2}$ The departmental protocol is that, in addition to sampling the tumour, one block is taken from each of the four quadrants and one block is taken vertically through the nipple for all mastectomy specimens. Blocks are of standard size; although large sections are used occasionally for histological examination of tumours, these are not used for random quadrant or nipple sampling.

All mastectomy specimens for carcinoma that were received in the histopathology department of Nottingham City Hospital, UK in the year 2003 were included in our study. Completion mastectomies, performed after previous diagnostic or therapeutic excisions, were included. Patients who received preoperative chemotherapy were excluded.

The number of quadrant blocks and nipple blocks from each of these cases was recorded. The presence of Paget's disease of the nipple was noted and any previous nipple biopsies were reviewed. All cases reported as showing multifocal disease were reviewed to determine whether the multifocal disease was detected on macroscopic examination or was only apparent on microscopic examination of tumour, quadrant, or nipple blocks. Multifocality was defined as more than one distinct focus of carcinoma, with at least $5 \mathrm{~mm}$ of

Abbreviations: DCIS, ductal carcinoma in situ; NHSBSP, National Health Service Breast Screening Programme 
intervening normal tissue. Our study was discussed with the chair of the hospital ethics committee and formal ethical approval was not considered necessary.

\section{RESULTS}

The total number of mastectomies performed for carcinoma, including completion mastectomies, received in the histopathology department of Nottingham City Hospital in the year 2003 was 248 . Review of these showed that in nine cases no nipple block was taken. Of these, two were subcutaneous mastectomies and in one the nipple had been removed by previous surgery. For the remaining cases there was no apparent reason for the nipple block not being taken.

Paget's disease of the nipple was seen in eight mastectomy specimens. In five cases, this was confirmation of a previous diagnosis made on biopsy of the nipple. In the remaining three, this was an unexpected diagnosis on examination of the nipple block of the mastectomy (in all three the nipple was macroscopically normal).

In 220 specimens all four quadrants were sampled. Three quadrant blocks were taken in nine cases, two blocks were taken in 10, one block in four, and no blocks in five. Of the 28 specimens with less than four quadrant sections, eight had a cavity from previous surgery, nine had a tumour larger than $30 \mathrm{~mm}$, and for the remaining 11 no reason could be identified.

In the 220 cases where all four quadrants were sampled, we identified 39 cases with multifocal disease. Multifocality was seen more often in specimens with invasive carcinoma ( 39 of $186 ; 21 \%$ ) than in specimens with only DCIS ( 0 of 34 ; $\left.0 \% ; \chi^{2}=7.3, p=0.007\right)$. Thus, for consideration of multifocality, the specimens were divided into those with invasive carcinoma and those with pure DCIS (table 1). There was no significant difference in the frequency of multifocal disease in conversion mastectomy specimens and mastectomies without previous surgery.

Of the 186 specimens with invasive carcinoma and sampling of all four quadrants, 20 cases were identified as multifocal on macroscopic examination of the specimen. In four cases, microscopy of the tumour blocks themselves revealed multifocal disease and the quadrant and nipple blocks did not provide additional information. Multifocality was unexpectedly discovered on microscopic examination of the quadrant sections in 11 cases, in the nipple alone in three, and in both the quadrant and nipple sections in one mastectomy specimen. The multifocal disease consisted of invasive carcinoma in 34 and both invasive and in situ carcinoma in five specimens. The multifocal disease was of similar morphology to the main tumour in 34 cases and of different appearance (histological grade or type) in five cases. Overall, multifocality was found on microscopic examination of quadrant or nipple sections in 15 of 220 (7\%) mastectomy specimens with all four quadrants sampled.

Table 1 also shows the rates of detection of multifocal disease in specimens with less than four quadrants sampled.

\section{DISCUSSION}

The main purpose of examination of histological sections of the nipple is the identification of Paget's disease. Paget's disease was identified in eight specimens (3\%), but this was a new diagnosis in only three $(1 \%)$. We are not aware of previous studies investigating the value of nipple sections. Although of interest, identification of Paget's disease in mastectomy specimens does not change patient management.

The main purpose of examination of quadrant sections is the identification of multifocal disease. Multifocal disease was identified in 39 of 220 (18\%) mastectomies in which all four quadrant sections were taken. However, about half of these were recognised on macroscopic examination (20 specimens) or on microscopy of the tumour sections (four specimens). Multifocal disease was unexpectedly found on microscopy of quadrant sections in only 12 cases $(5 \%)$, with three additional cases found on microscopy of the nipple section. Multifocality was seen more often in specimens with invasive carcinoma $(21 \%)$ than in specimens with only DCIS $(0 \%)$.

\section{"Although of interest, identification of Paget's disease in mastectomy specimens does not change patient manage- ment $^{\prime \prime}$}

The only previous similar study, by Gupta et al, found multifocal or multicentric disease in quadrant sections in 20 of 78 mastectomy specimens $(26 \%) .{ }^{4}$ Cases with macroscopically identified multifocal disease were excluded, so the difference between that study and our present study is even larger. Potential explanations for the higher rate of multifocality in Gupta's study include the higher number of quadrant blocks taken (range, 4-17; mean, 9) and the relative inexperience of the dissectors (half the specimens were examined by first year residents), who may not have identified some macroscopic abnormalities. In the present series, the macroscopic examination was undertaken by consultant breast histopathologists, histopathology trainees, and a specially trained biomedical scientist. The quadrant sections taken are not truly "random"; targeting of firmer areas of breast tissue is performed, so the macroscopic examination is important.

Although identification of multifocal disease in mastectomy specimens does not change patient management, confirmation of this finding may be reassuring for women

Table 1 Frequency of detection of multifocal disease, according to the number of quadrants sampled and whether there was invasive carcinoma or pure DCIS

\begin{tabular}{|c|c|c|c|c|c|c|}
\hline \multirow[b]{3}{*}{ Method of detection of multifocal disease } & \multicolumn{6}{|c|}{ Number of quadrants sampled } \\
\hline & \multicolumn{3}{|c|}{ Four quadrants } & \multicolumn{3}{|c|}{ Less than four quadrants } \\
\hline & Invasive & DCIS & Total & Invasive & DCIS & Total \\
\hline Macroscopic examination & 20 & 0 & 20 & 2 & 0 & 2 \\
\hline Microscopy of quadrant sections & 11 & 0 & 11 & 5 & 1 & 6 \\
\hline Microscopy of nipple sections & 3 & 0 & 3 & 0 & 0 & 0 \\
\hline Microscopy of nipple and quadrant sections & 1 & 0 & 1 & 0 & 0 & 0 \\
\hline Microscopy of tumour sections (satellite foci near main lesion) & 4 & 0 & 4 & 0 & 0 & 0 \\
\hline Total with multifocal disease & 39 & 0 & 39 & 7 & 1 & 8 \\
\hline Total number of specimens & 186 & 34 & 220 & 20 & 8 & 28 \\
\hline
\end{tabular}




\section{Take home messages}

- Careful macroscopic examination of mastectomy specimens and sampling of abnormal areas is important because about half of the cases of multifocal disease are identified this way

- Although additional sections of nipple and random quadrant sections should ideally be taken, unexpected Paget's disease or multifocal disease will be found only in a small number of specimens

- Therefore, these sections should not be mandatory and should be taken only if resources permit

who had a mastectomy performed because of the preoperative diagnosis of multifocal disease, and for those who had had difficulty in selecting the type of surgery (wide local excision or mastectomy) and who had chosen mastectomy.

Our study suggests that the careful macroscopic examination of mastectomy specimens and sampling of abnormal areas is important; half of the cases of multifocal disease are identified macroscopically. Additional sections of nipple and random quadrant sections in mastectomies performed for cancer should ideally be taken, but such sections will show unexpected Paget's disease or multifocal disease in only a small number of specimens. This suggests that these sections should not be mandatory and should be taken only if resources permit.

\section{Authors' affiliations}

K Sikand, A H S Lee, S E Pinder, C W Elston, I O Ellis, Department of Histopathology, City Hospital, Hucknall Road, Nottingham NG5 1PB, UK

\section{REFERENCES}

1 Rosen PP. Rosen's breast pathology, 2nd ed. Philadelphia: Lippincott, Williams and Williams, 2001.

2 National coordinating group for breast screening pathology. Pathology reporting in breast cancer screening, 2nd ed. Sheffield: National Health Service Breast Screening Programme, 1995.

3 The UK national coordinating committee for breast screening pathology Pathology reporting of breast disease. Sheffield: National Health Service Breast Screening Programme and the Royal College of Pathologists, 2005.

4 Gupta D, Nath M, Layfield U. Utility of four-quadrant random sections in mastectomy specimens. Breast J 2003;9:307-11. 